\title{
Usage of the influence coefficient during calculation of a nominal error of gas counters
}

\author{
M.S. Ostapenko ${ }^{1, *}$, M.A. Popova ${ }^{1}$, and A.M. Tveryakov ${ }^{1}$ \\ ${ }^{1}$ Tyumen industrial university, Tyumen, Russia
}

\begin{abstract}
In this paper, we evaluate the method of finding the relative error of gas flow meters taking into account the influence coefficients. A literature analysis was carried out, which showed that flow meters are used at oil and gas enterprises, which show its metrological characteristic, showing specific values of gas flow in operating conditions. Various types of gas flow meters are considered, with a description of the quality indicators of the devices. An additional error was investigated depending on changes in operating conditions. The calculations of the relative error of the meter taking into account the limiting values of the additional errors indicated in the technical documentation, as well as calculations taking into account the coefficients of influence under operating conditions. Based on the obtained values of the influence coefficients, graphs were constructed on which the effect of temperature and pressure on the error was determined. The article provides tabular values of the influence coefficients for petroleum gas, a conclusion is drawn on the applicability of this method.Oil and gas industry have a great influence on development of national economy in our country. Oil and gas have a leading position in energy industry and they are more effective and energy-intense in comparison with other natural substances.
\end{abstract}

Oil gas which is a high-grade stock is used in many production facilities of chemical industry. At such production facilities gas (which is a natural component) consumption metering relates to priority tasks of the production facility. Flow meters are used, which show concrete values of gas spending in operating conditions due to their metrological characteristics in order to know the exact values of oil gas spending.

Therefore the spending value shown by the counter is an important sphere for a study because the spending value depends on several influencing factors at once (environmental and process temperature, the pressure inside of the pipeline system, volume components of gas and other)

Nowadays this theme is essential because sharp increase of demand for energy sources and of their cost happens and that is the reason why each consumer wants to know about his/her spending of resources for cash savings.

There are many devices which measure spending and quantity of a substance. They differ from each other according to a type of functioning and a method of measurement.

* Corresponding author: ms_ostapenko@mail.ru 
When choosing a device for spending measurement, specialists take into account the substance features, parameters and precision of measurement. It follows that resources metering accuracy becomes of a high priority.

In this article we mention gas as a target medium because only gas flow meters are studied. There are different types of gas flow meters: vortex, electromagnetic, ultrasonic and others. All gas flow meters are meant for reaching of the following purposes:

- Receiving of a basis for settlements between a supplier, a pipeline organization, a gas distribution organization and a buyer (consumer) of gas in accordance with agreements on supply and provision of services of gas transportation;

- An analysis of management of gas supply and transportation, and also optimization of these processes;

- Provision of a balanced system of gas supply system during gas distribution and transportation;

- Control over rational and effective gas usage.

Nowadays there is a struggle for a leading place on the market among facilities producing counters for fiscal metering of gas. Increase of production quality is an integral part of any facility producing one or another device. Facilities continuously aim at improvement of quality characteristics of their production in order not only to hold at the market but also to outdistance competitors. We can refer a device accuracy to quality characteristics because this device involves high quality.

All facilities aim at improvement of accuracy characteristics of their products. In order to obtain an ultimate value of the nominal error in the technical documentation of the counters most of facilities normalize ultimate values of additional errors caused by a change of influencing factors (for example of temperature and pressure of the process). It means that the error value that we obtain under operational conditions must not exceed the ultimate value of the error stated in the technical documentation [1,2].

In most cases the counter is a complex of measurement means which consists from a gas-consumption gauge, a temperature sensor, a pressure sensor and a computing machine. It follows that the nominal error of the counter is caused by nominal errors of its parts, i.e. the consumption gauge, the pressure sensor, temperature sensor and the computing machine. Therefore under operating conditions the nominal errors dynamics of measurement devices included as a compound of the counter can have a big influence on the nominal error of the counter.

In case of a change of conditions under which gas consumption measurement also values of the component errors, therefore the nominal error margin of the counter changes in general. In other words additional errors appear because of a change of the device operating conditions (in this work: the temperature and pressure of the target medium).

According to the technical documentation of the gas flow counters a consumer knows the ultimate value of the nominal error of the gas flow counter but under the operating conditions the true value of the error can differ from its ultimate value, stated in the technical documentation.

That is why it makes sense to estimate and use so called influence functions in order to estimate the nominal error. The influence function - is a dependence of a change of metrological characteristics of a measurement device on a change of an influence quantity. According to AUSS (All-Union State Standard) 8.009 [3] the influence function can be presented as a number, formula, table or a diagram. Also it is mentioned in AUSS 8.009 that the influence quantity which passes through the origin can be presented as an influence coefficient numerically. The first official influence coefficient was offered by Lionell Penrouse in 1946, though such idea was proposed by Martin Luther yet in 1787 .

In this article we used the influence coefficients as the influence function in allowance of linearity of contributing factors influence. The influence coefficient shows how the value 
received at the end of the process changes. In this case the temperature and pressure of the process will be the influence values. This method of calculation consists of that the influence coefficient (the value on the influence curve) is input in the nominal error calculation formula.

The influence coefficients are calculated according to the formulas:

$$
\zeta_{T}=\frac{V_{T 1}-V_{T 2}}{\Delta T} \frac{T}{V_{T 2}}
$$

where, $V_{T 1}=$ is a volume under standard conditions under the temperature increased by the value of the absolute error of measurement of temperature $\Delta \mathrm{T}$ (i.e. $\mathrm{T}+\Delta \mathrm{T}$ ); $V_{T 2}=$ a volume under standard conditions under the period average temperature value of the measurable gas $\mathrm{T} ; T=$ the temperature of the measurable gas; $\Delta T=$ absolute error of the temperature measurement.

$$
\zeta_{P}=\frac{V_{P 1}{ }^{-V_{P 2}}}{\Delta P} \frac{P}{V_{P 2}}
$$

where, $V_{P 1}=$ is a volume under standard conditions under the pressure increased by the value of the absolute error of measurement of pressure $\triangle P ; V_{P 2}-$ a volume under standard conditions under the period average temperature value of the measurable gas; $P$ - the pressure of the measurable gas; $\Delta P$ - absolute error of the pressure measurement.

$V_{T 1}$ and $V_{P 1}$ are values of the quoted gas $\left(\mathrm{V}^{\prime}\right)$, which is calculated according to the following formula:

$$
V^{\prime}=2,89 \frac{P_{W C}}{T_{W C}{ }{ }_{\text {compr }}}
$$

where, $P_{W C}=$ absolute pressure under working conditions $(\mathrm{Kpa}) ; T_{W C}=$ process temperature under working conditions $(\mathrm{K}) ; K_{\text {compr }}=$ compressibility coefficient.

In turn the compressibility coefficient expresses deviation of the real gas properties from the ideal gas properties. This deviation is found in case if the obtained consumption is necessary to correct to the standard conditions. The methods of finding of the compressibility for oil gas is described in Standard reference data on physical constants and properties of substances and materials (SSRDS) 113. This methods was used for the calculations. Besides the oil gas there is a natural gas, abstinently compressed gas mixtures, nitro and others. For each of targets medium their own compressibility coefficients are found according to the methods described in AUSS 30319.2, SSRDS 118, SSRDS 228 $[4,5,6]$. At one of instrument-making facilities of Tyumen region program "Phisics" is developed with the purpose of time saving and exclusion of mistakes during the calculation of the compressibility coefficient (i.e. exclusion of a human factor). The abovementioned methods are released within the frameworks of this program in order to calculate parameters of measured gases.

Since the error is influence by the process temperature and pressure change than the coefficients of influence from the temperature change $\left(\zeta_{T}\right)$ under different pressures and from the pressure change $\left(\zeta_{P}\right)$ under different temperatures were calculated. The oil gas which exists under conditions from $-10^{\circ} \mathrm{C}$ to $+150^{\circ} \mathrm{C}$ and from $100 \mathrm{Kpa}$ to $10000 \mathrm{Kpa}$ was chosen as the target medium. The obtained values of the coefficients are stated in tables 1 and 2. 
Table 1. The dependence of the influence coefficient $\left(\zeta_{T}\right)$ from the temperature under different pressures.

\begin{tabular}{|c|c|c|c|c|c|c|c|c|c|c|c|c|}
\hline $\begin{array}{c}\text { P, Kpa } \\
\mathbf{T},{ }^{\circ} \mathbf{C}\end{array}$ & $\mathbf{1 0 0}$ & $\mathbf{2 0 0}$ & $\mathbf{3 0 0}$ & $\mathbf{4 0 0}$ & $\mathbf{5 0 0}$ & $\mathbf{1 0 0 0}$ & $\mathbf{2 0 0 0}$ & $\mathbf{3 0 0 0}$ & $\mathbf{4 0 0 0}$ & $\mathbf{5 0 0 0}$ & $\mathbf{7 5 0 0}$ & $\mathbf{1 0 0 0 0}$ \\
\hline $\mathbf{- 1 0}$ & $-1,010$ & $-1,023$ & $-1,035$ & $-1,048$ & $-1,062$ & $-1,133$ & $-1,309$ & $-1,550$ & $-1,916$ & $-2,564$ & & \\
\hline $\mathbf{0}$ & $-1,009$ & $-1,021$ & $-1,032$ & $-1,043$ & $-1,055$ & $-1,119$ & $-1,270$ & $-1,468$ & $-1,745$ & $-2,167$ & & \\
\hline $\mathbf{1 0}$ & $-1,008$ & $-1,018$ & $-1,028$ & $-1,039$ & $-1,049$ & $-1,105$ & $-1,234$ & $-1,396$ & $-1,607$ & $-1,897$ & $-3,828$ & \\
\hline $\mathbf{2 0}$ & $-1,008$ & $-1,016$ & $-1,026$ & $-1,035$ & $-1,045$ & $-1,096$ & $-1,211$ & $-1,354$ & $-1,530$ & $-1,762$ & $-2,937$ & \\
\hline $\mathbf{4 0}$ & $-1,006$ & $-1,013$ & $-1,022$ & $-1,029$ & $-1,037$ & $-1,079$ & $-1,170$ & $-1,276$ & $-1,401$ & $-1,553$ & $-2,119$ & $-3,476$ \\
\hline $\mathbf{6 0}$ & $-1,004$ & $-1,012$ & $-1,018$ & $-1,024$ & $-1,031$ & $-1,065$ & $-1,139$ & $-1,222$ & $-1,317$ & $-1,423$ & $-1,773$ & $-2,342$ \\
\hline $\mathbf{8 0}$ & $-1,004$ & $-1,009$ & $-1,015$ & $-1,021$ & $-1,026$ & $-1,055$ & $-1,116$ & $-1,182$ & $-1,255$ & $-1,336$ & $-1,577$ & $-1,909$ \\
\hline $\mathbf{1 0 0}$ & $-1,003$ & $-1,008$ & $-1,012$ & $-1,017$ & $-1,022$ & $-1,047$ & $-1,097$ & $-1,152$ & $-1,210$ & $-1,272$ & $-1,452$ & $-1,673$ \\
\hline $\mathbf{1 2 0}$ & $-1,003$ & $-1,007$ & $-1,011$ & $-1,015$ & $-1,019$ & $-1,040$ & $-1,083$ & $-1,129$ & $-1,176$ & $-1,226$ & $-1,363$ & $-1,525$ \\
\hline $\mathbf{1 4 0}$ & $-1,003$ & $-1,006$ & $-1,010$ & $-1,013$ & $-1,016$ & $-1,034$ & $-1,070$ & $-1,109$ & $-1,148$ & $-1,189$ & $-1,300$ & $-1,422$ \\
\hline $\mathbf{1 5 0}$ & $-1,002$ & $-1,006$ & $-1,009$ & $-1,011$ & $-1,015$ & $-1,032$ & $-1,066$ & $-1,100$ & $-1,137$ & $-1,174$ & $-1,274$ & $-1,382$ \\
\hline
\end{tabular}

Table 2. The dependence of the influence coefficient $\left(\zeta_{P}\right)$ from the pressure under different temperatures.

\begin{tabular}{|c|c|c|c|c|c|c|c|c|c|c|c|c|}
\hline $\begin{array}{c}\text { P, Kpa } \\
\mathbf{T},{ }^{\circ} \mathbf{C}\end{array}$ & $\mathbf{1 0 0}$ & $\mathbf{2 0 0}$ & $\mathbf{3 0 0}$ & $\mathbf{4 0 0}$ & $\mathbf{5 0 0}$ & $\mathbf{1 0 0 0}$ & $\mathbf{2 0 0 0}$ & $\mathbf{3 0 0 0}$ & $\mathbf{4 0 0 0}$ & $\mathbf{5 0 0 0}$ & $\mathbf{7 5 0 0}$ & $\mathbf{1 0 0 0 0}$ \\
\hline $\mathbf{- 1 0}$ & 1,008 & 1,015 & 1,023 & 1,031 & 1,040 & 1,085 & 1,195 & 1,343 & 1,576 & 1,982 & & \\
\hline $\mathbf{0}$ & 1,007 & 1,013 & 1,021 & 1,028 & 1,035 & 1,074 & 1,168 & 1,290 & 1,454 & 1,718 & & \\
\hline $\mathbf{1 0}$ & 1,003 & 1,006 & 1,008 & 1,011 & 1,016 & 1,031 & 1,064 & 1,132 & 1,184 & 1,278 & 1,874 & \\
\hline $\mathbf{2 0}$ & 1,005 & 1,010 & 1,016 & 1,022 & 1,027 & 1,055 & 1,127 & 1,203 & 1,308 & 1,436 & 2,141 & \\
\hline $\mathbf{4 0}$ & 1,004 & 1,008 & 1,013 & 1,017 & 1,021 & 1,043 & 1,096 & 1,154 & 1,228 & 1,305 & 1,610 & 2,374 \\
\hline $\mathbf{6 0}$ & 1,003 & 1,006 & 1,010 & 1,014 & 1,017 & 1,035 & 1,074 & 1,114 & 1,163 & 1,208 & 1,384 & 1,677 \\
\hline $\mathbf{8 0}$ & 1,003 & 1,005 & 1,008 & 1,010 & 1,013 & 1,026 & 1,057 & 1,087 & 1,127 & 1,161 & 1,268 & 1,423 \\
\hline $\mathbf{1 0 0}$ & 1,002 & 1,004 & 1,006 & 1,008 & 1,010 & 1,020 & 1,045 & 1,068 & 1,092 & 1,126 & 1,196 & 1,292 \\
\hline $\mathbf{1 2 0}$ & 1,002 & 1,003 & 1,004 & 1,006 & 1,008 & 1,016 & 1,032 & 1,049 & 1,066 & 1,083 & 1,144 & 1,196 \\
\hline $\mathbf{1 4 0}$ & 1,001 & 1,002 & 1,004 & 1,005 & 1,006 & 1,010 & 1,024 & 1,037 & 1,049 & 1,062 & 1,094 & 1,149 \\
\hline $\mathbf{1 5 0}$ & 1,001 & 1,002 & 1,003 & 1,004 & 1,005 & 1,010 & 1,020 & 1,036 & 1,041 & 1,061 & 1,078 & 1,126 \\
\hline
\end{tabular}

On the obtained values of the influence coefficient diagrams were constructed for display purposes. On these diagrams we can see influence of the temperature and pressure on the error.

In order to make an assessment of this method we made calculation of the nominal error of the counter taking into account the ultimate values of additional errors stated in its technical documentation and also calculations involving the influence coefficients under the operation conditions. In this case as a result we will have the nominal error of the counter and the contribution errors will be entry.

Calculations of the nominal error were made by means of two formulas:

$$
\delta_{V 1}^{S C}= \pm \sqrt{\delta_{V}^{2}+\delta_{\text {add }}^{T}{ }^{2}+\delta_{\text {add }}^{P}{ }^{2}+\delta_{T}^{2}+\delta_{P}^{2}+\delta_{\text {calc }}^{2}}
$$

where, $\delta_{V}=$ limits of the permissible nominal error of the counter caused by the error; $\delta^{T}$ add $=$ limits of the permissible additional error from the temperature change; $\delta^{P}$ add $=$ limits of the permissible additional error of the counter because of the change of the process 
pressure; $\delta_{T}=$ Limits of the permissible nominal error of the counter caused by the error of the temperature measurement; $\delta_{P}=$ limits of the permissible nominal error of the counter

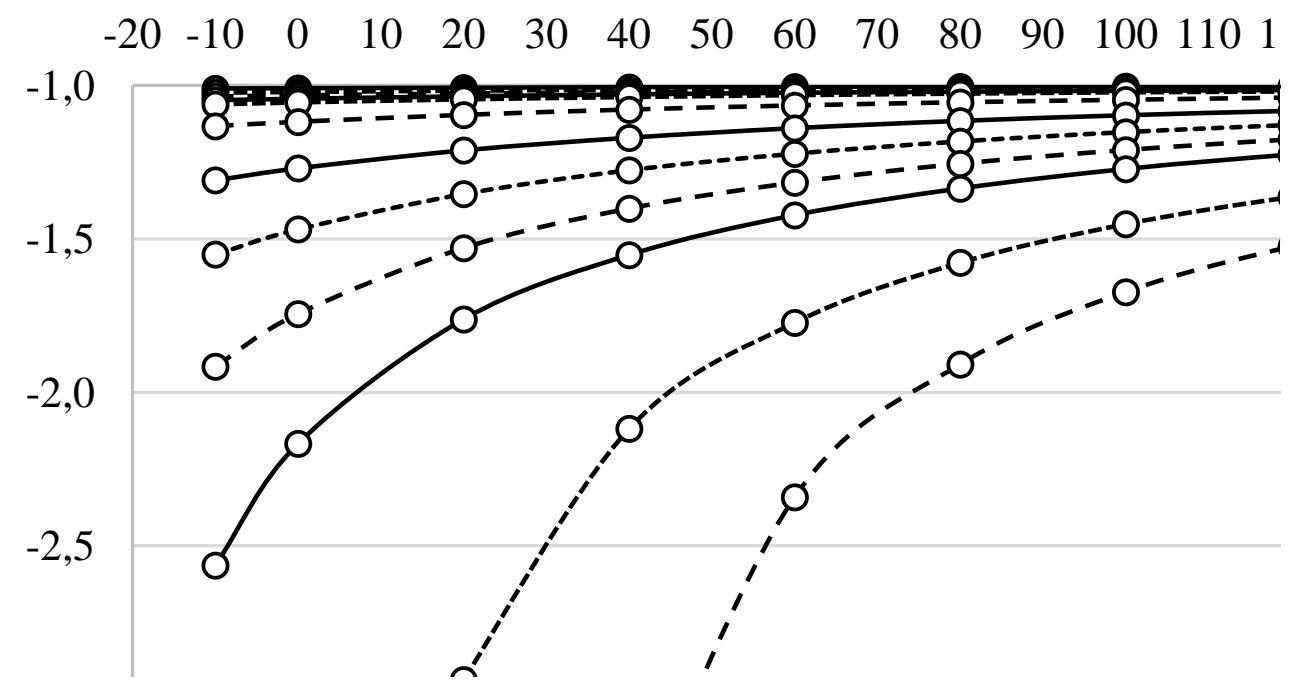

Fig. 1. The diagram of the dependence of the influence coefficient $\left(\zeta_{T}\right)$ from the temperature under different pressures $(P)$

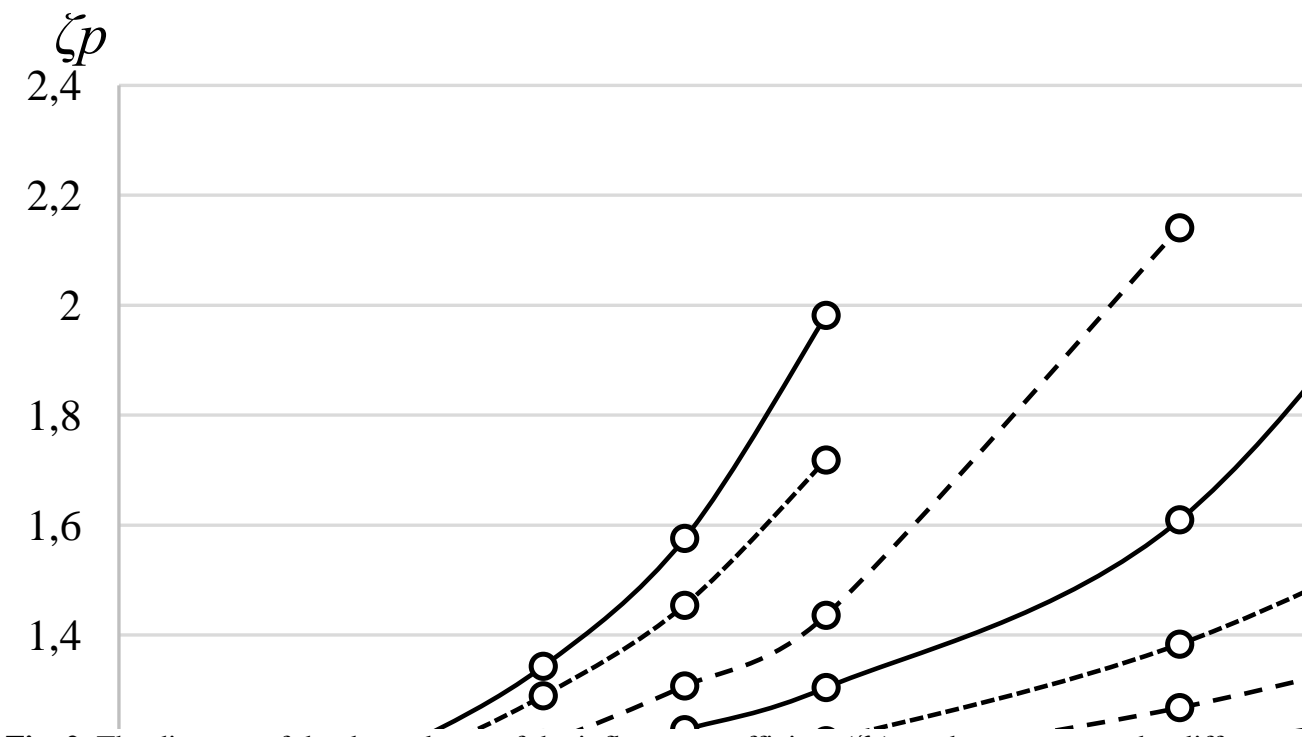

Fig. 2. The diagram of the dependence of the influence coefficient $\left(\bar{\zeta}_{P}\right)$ on the pressure under different temperatures $(T)$.

caused by the error of the pressure measurement; $\delta_{\text {calc }}=$ limits of the permissible nominal error of the counter caused by the errors of the volume calculation corrected to the standard conditions.

$$
\delta_{V 1}^{S C}= \pm \sqrt{\delta_{V}^{2}+\left(\zeta_{T}+\delta_{T}\right)^{2}+\left(\zeta_{P}+\delta_{P}\right)^{2}+\delta_{\text {calc }}^{2}}
$$


where $\zeta_{T}=$ the coefficient of influence of the temperature change on the error; $\zeta_{P}=$ the coefficient of influence of change of the pressure on the error.

As a result of the conducted calculations the following errors values were obtained. Part of them is stated in table 3 .

Table 3. Results of the error calculation

\begin{tabular}{|l|c|c|}
\hline $\begin{array}{l}\text { Conditions (temperature, } \\
\text { pressure) }\end{array}$ & $\delta_{V 1}^{S C}, \%$ & $\delta_{V 2}^{S C}, \%$ \\
\hline $\begin{array}{l}T=+10^{\circ} \mathrm{C} \\
P=200 \mathrm{Kpa}\end{array}$ & 1.61 & 1.60 \\
\hline $\begin{array}{l}T=+40^{\circ} \mathrm{C} \\
P=500 \mathrm{Kpa}\end{array}$ & 1.71 & 1.68 \\
\hline $\begin{array}{l}T=+60^{\circ} \mathrm{C} \\
P=500 \mathrm{Kpa}\end{array}$ & 2.89 & 2.82 \\
\hline
\end{tabular}

From the obtained calculated data we can draw a conclusion that this method of the error assessment is useful for finding of a real value of the basic nominal error under operating conditions of the gas flow counter since the obtained value of the error does not exceed the permissible value of the nominal error.

This method of the nominal error assessment under the operating conditions will be very convenience for consumers who want to know the real value of the consumed gas with the purpose of financial resources saving.

\section{References}

1. Bolarev B.P., Standardization metrology, confirmation of correspodence (RDE INFRA, Moscow,2013)

2. Shishkin I.F., Theoretical metrology (St. Pete, Saint-Petersburg,2010)

3. AUSS 8.009-84 State system of provision of uniformity of measurements - State system of ensuring uniform measurement. Normalized metrological characteristics of measurement tools.

4. AUSS 30319.2-2015 Natural gas. Methods of calculation of physical characteristic. Calculation of physical properties on the basis of data about density under standard conditions and undernitro carbon dioxide.

5. SSRDS 118-2005 Methods of SSRDS. Calculation of density, compressibility factor, value of adiabat and coefficient of abstinently compressed gas mixtures.

6. SSRDS 228-2014 - Methods of calculation determination of thermodynamic properties, coefficient of dynamic coefficient of viscosity. 